\title{
Joint theoretical and experimental study on elastic electron scattering from bismuth
}

\author{
B. Predojević, ${ }^{*}$ D. Šević $\odot$, and B. P. Marinković $\odot$ \\ Institute of Physics Belgrade, University of Belgrade, Pregrevica 118, 11080 Belgrade, Serbia \\ R. P. McEachran \\ Plasma Research Laboratories, The Research School of Physics, Australian National University, Canberra, ACT 0200, Australia \\ F. Blanco $\odot$ \\ Departamento de Estructura de la Materia, Física Térmica y Electrónica, Universidad Complutense de Madrid, \\ Avenida Complutense, E-28040, Madrid, Spain \\ G. García \\ Instituto de Física Fundamental, CSIC, Serrano 113-bis, E-28006, Madrid, Spain \\ M. J. Brunger $\oplus^{\dagger}$ \\ College of Science and Engineering, Flinders University, GPO Box 2100, Adelaide, South Australia 5001, Australia \\ and Department of Actuarial Science and Applied Statistics, Faculty of Business and Information Science UCSI, \\ Kuala Lumpur 56000, Malaysia
}

(Received 28 November 2019; revised manuscript received 28 January 2020; accepted 25 February 2020; published 23 March 2020)

\begin{abstract}
We report on experimental elastic differential and integral cross sections for electron scattering from bismuth. The energy range of those measurements is $10-100 \mathrm{eV}$, while the scattered electron angular range in the differential cross section data is $10^{\circ}-150^{\circ}$. We also supplement our experimental results with theoretical data from our optical potential approach, in this case applying two different sets of scattering potentials to the problem. Good overall qualitative accord is typically observed between our measured and calculated results, particularly when allowance is made for the dimer contribution in the experimental atomic beam.
\end{abstract}

DOI: 10.1103/PhysRevA.101.032704

\section{INTRODUCTION}

Interest in studying the properties of bismuth $(\mathrm{Bi})$ arises from both fundamental and applied reasons. From a fundamental perspective $\mathrm{Bi}$ is a heavy atom so that relativistic effects in the electron-scattering process might be anticipated. Indeed both measurements and calculations that look at the polarization of elastically scattered electrons, thereby allowing a detailed study of spin-dependent interactions during the scattering process, have been undertaken [1,2]. Additionally, with its open shell structure (a ground-state configuration of $[\mathrm{Hg}] 6 \mathrm{p}^{3}{ }^{4} S_{3 / 2}$ ), Bi represents a stringent test in describing both its valence-electronic structure and then employing the results of those structure calculations in electron-scattering theoretical computations [3]. The presence of bismuth lines in stellar spectra has been reported in several different types of chemically peculiar stars [4-6]. Quantitative modeling of those spectra require $[7,8]$, in part, both electron- and photondriven cross sections, transition probabilities, astrochemical

\footnotetext{
*Faculty of Natural Sciences, University of Banja Luka, Republic of Srpska, Bosnia and Herzegovina.

${ }^{\dagger}$ Corresponding author: michael.brunger@flinders.edu.au
}

reaction rates, and so on. This modeling can in turn lead to remote sensing opportunities being uncovered, such as described in past work by Campbell and colleagues [9-11]. From an applied perspective, bismuth has also generated some interest in the field of nanotechnology $[12,13]$.

There is currently in the literature only a few available papers containing results for calculations of differential cross sections (DCSs) for elastic electron scattering from the bismuth atom. Schonfelder [14] used a relativistic Hartree approximation to calculate an elastic DCS at $100 \mathrm{eV}$ incident electron energy, while Fink et al. [15] and Fink and Yates [16] solved the Dirac equation, with a Kohn-Sham representation for the target states, to determine the elastic DCS in the $100-1500 \mathrm{eV}$ energy range. Somewhat more recently, Neerja and Tripathi [3] employed a semirelativistic approach, in the energy range $2-500 \mathrm{eV}$, in order to report elastic DCSs, elastic integral cross sections (ICSs), and momentum transfer cross sections (MTCSs). Given this sparsity of available theoretical results, one rationale behind this investigation was to employ our optical potential (OP) approach [17] to this scattering system in order to at least partially alleviate that deficiency. From an experimental perspective, the first measured data were the relative elastic angular distributions of Haug [18]. That work was undertaken over the scattered electron angular range 
$\theta_{\mathrm{e}}=30^{\circ}-155^{\circ}$, and for incident electron energies between 5-1200 eV. The only absolute elastic DCS measurement currently available is from Williams et al. [19], at the single incident electron energy of $40 \mathrm{eV}$ and for $\theta_{\mathrm{e}}=10^{\circ}-130^{\circ}$. It is understood in the electron-scattering community that the ground-breaking electron-metal vapor measurements, made at the Jet Propulsion Laboratory (JPL) from the early 1970s1980s, for both elastic and discrete inelastic processes, have not stood the test of time and are inaccurate. There are many examples confirming that assertion, including for sodium [20], magnesium [21,22], and zinc [23]. Thus a further rationale behind this submission was to check that scenario explicitly for bismuth [19] and, just as importantly, to extend the available experimental elastic cross section data base beyond the single 40-eV measurement. This is absolutely crucial in terms of providing a serious benchmark to test theory against. For completeness, we also note the work from the Belgrade group on electron impact excitation of the inelastic $6 \mathrm{p}^{2} 7 \mathrm{~s}{ }^{4} P_{1 / 2}$ level of bismuth [24] and on its autoionizing states [25].

The remainder of this paper is structured as follows. In Sec. II we present a brief discussion of our experimental methodology, while in Sec. III a précis of our OP method is provided. Our results and discussion are given in Sec. IV, with some conclusions from this study thereafter being drawn.

\section{EXPERIMENTAL CONSIDERATIONS}

The apparatus and measurement procedures for this study are very similar to those we employed in our recent work with zinc [23], and so only a brief précis of the features specific to bismuth need to be addressed here. A conventional crossed-beam electron spectrometer and atomic-beam source was employed, with both the electron beam and atomic beam being highly stable (to better than $\sim 2 \%$ in each case) throughout our experiments.

The incident electron energies $\left(E_{0}\right)$ in this investigation were $10,20,40,60,80$, or $100 \mathrm{eV}$, with typical electron-beam currents in the range 1-10 nA, as measured with a standard Faraday Cup configuration, being achieved. The overall energy resolution was $\sim 150 \mathrm{meV}$ full width at half maximum (FWHM), while the incident electron-beam energy scale was calibrated against the well-known $3 \mathrm{~s} 3 \mathrm{p}{ }^{1} P$ excitation threshold of magnesium at $4.346 \mathrm{eV}$ and is accurate to $\sim \pm 300 \mathrm{meV}$ (FWHM). The angular resolution of the spectrometer was estimated to be $1.5^{\circ}$ (FWMH), while the uncertainty in the angular scale, the true zero-degree scattering angle $\left(\theta_{\mathrm{e}}\right)$ being established by the symmetry of the elastic intensity between $-20^{\circ}$ and $+20^{\circ}$, is $\pm 0.5^{\circ}$. Note that the scattered electron angular range of the present DCSs was $10^{\circ}-150^{\circ}$, with the minimum angle being set by primary electron-beam interference and the maximum angle being defined by the physical sizes of the electron monochromator and electron analyzer.

The bismuth atomic beam, formed from pure $\mathrm{Bi}$ granules, was generated using a resistively heated oven fabricated from titanium. Note that water cooling of the oven shield, in conjunction with thermal insulation from ceramic components, minimized the potential effects of the high oven temperature $(T)$ on all components of the apparatus including the channel electron multiplier detector. The oven nozzle aspect ratio was 0.075 , a small enough value that should assist in minimizing any possible effective-path-length correction factor effects on the measured angular distributions even for a single-tube capillary such as here [26,27] (see below). Monitoring of the oven temperature was crucial to ensure a stable atomic beam, with a somewhat higher temperature at the top of the crucible ensuring the nozzle did not clog and a somewhat lower $T(\sim 1000 \mathrm{~K})$ at the bottom providing the effusive flow of the Bi beam. The corresponding metal vapor pressure $(P)$ was approximately $10 \mathrm{~Pa}$, while the background pressure in the chamber was better than $5 \mathrm{mPa}$. Under those operating conditions $(T, P)$ bismuth is known to cluster [28], with an estimate of $80 \%$ monomer and $20 \%$ dimer being determined here. In 1963 Nesmeyanov [28] published a book in which he reviewed the variation of vapor pressure of different species (atoms and molecules) with temperature including their cluster compositions. For bismuth his review covered several ranges of temperature, overall from 480 to $2500 \mathrm{~K}$, while the dimer percentage was determined by means of mass spectrometry analyses. Nesmeyanov [28] started with the paper of Barus [29] and then covered several different methods for elucidating the dependence of $P$ on $T$ including the Knudson effusion method [30] and Langmuir vaporization technique for liquid phase vapor pressure and partial pressure measurements [31]. He obtained the following relation:

$$
\log (P)=A-B / T+C \times T+D \log (T),
$$

where $T$ is in Kelvin and $P$ in Torr and the coefficients are determined for liquid bismuth as $A=11.48875 ; B=$ 9217.28; $C=-0.000061149$; and $D=0.95933$. In his book the relevant table presents, as a function of temperature, the total $\mathrm{Bi}$ vapor pressure, atomic partial pressure $\left(P_{a}\right)$, and dimer partial pressure $\left(P_{m}\right)$. For a temperature $\sim 1000 \mathrm{~K}$, and by applying gas kinetic theory, the ratio of the number density of dimers $\left(n_{m}\right)$ to the total number density $(n)$, as given by

$$
n_{m} / n=1 /\left(1+\left(\frac{P_{a}}{P_{m}}\right)\right),
$$

which leads to a value of $n_{m} / n \sim 0.20$ with the values of $P_{a}=$ 0.216 Torr and $P_{m}=0.055$ Torr. The estimated uncertainty on that value of $n_{m} / n$ depends on the uncertainty in the model $P$ versus $T$ curve and the uncertainty on our measured value of $T$. Here this overall uncertainty was thought to be of the order of $10 \%$, giving the value of $n_{m} / n=0.20 \pm 0.02$. Note that this estimate is consistent with the $15 \%-30 \%$ dimer contribution from Kaussen et al. [1] at their higher working temperature of $1500 \mathrm{~K}$. Further note that this is a crucial point to remember when we compare our theoretical and experimental cross section results in Sec. IV.

After appropriate background subtraction, two approaches, which gave self-consistent results for the derived DCSs at each $E_{0}$ and $\theta_{\mathrm{e}}$, were employed to set the absolute scale of our data [23]. In the first normalization approach, for a given $E_{0}$, the elastic angular distributions were determined by measuring the scattered elastic count rate (i.e., at $0 \mathrm{eV}$ energy loss) at each chosen scattered electron angle $\theta_{\mathrm{e}}$, and then correcting those data for the forward angle scattering effect using the approach of Brinkman and Tramar [26]. This angular distribution (elastic counts versus $\theta_{\mathrm{e}}$ ) was subsequently placed on an absolute scale, from energy loss measurements 

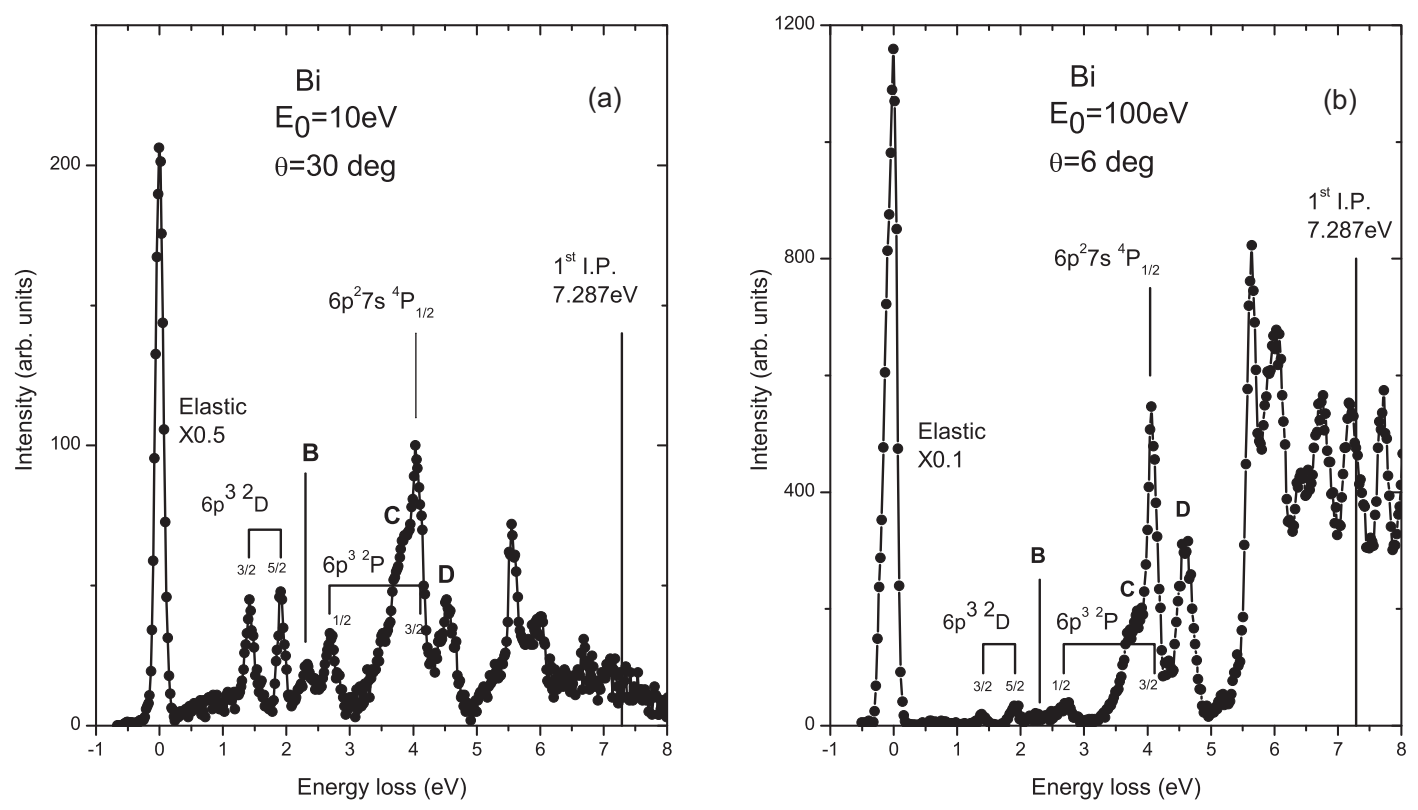

FIG. 1. Typical electron energy-loss spectra for electrons scattering from Bi. The relevant kinematical conditions are as follows: (a) $E_{0}=$ $10 \mathrm{eV}$ and $\theta_{\mathrm{e}}=30^{\circ}$ and (b) $E_{0}=100 \mathrm{eV}$ and $\theta_{\mathrm{e}}=6^{\circ}$. The three features that correspond to excitations of the molecular states of $\mathrm{Bi}_{2}$ are labeled B, C, D.

(see Fig. 1) that encompassed the elastic $\left({ }^{4} S_{3 / 2}\right.$ state) and inelastic $\left(6 \mathrm{p}^{2} 7 s{ }^{4} P_{1 / 2}\right)$ peaks, at one or two specific normalization angles. This approach, of sitting on the elastic peak and recording the count rate as a function of $\theta_{\mathrm{e}}$, is relatively quick as only 1 or 2 cross-check energy loss spectra are then subsequently needed to set the absolute scale. From the ratio of the elastic to inelastic intensities, and a knowledge of the absolute ${ }^{4} P_{1 / 2}$ DCSs from Marinković et al. [24], our angular distribution measurements at the given energy could now be placed on an absolute scale. The only concern with this method, particularly at the smaller incident electron energies, is the behavior of the analyzer transmission as a function of the scattered electron energies. For example, this follows as for a $10-\mathrm{eV}$ incident electron the outgoing scattered electron energies will vary from $10 \mathrm{eV}\left({ }^{4} S_{3 / 2}\right.$ state $)$ to $6 \mathrm{eV}\left({ }^{4} P_{1 / 2}\right.$ state) across our energy-loss spectrum. However, our analyzer electron optics were designed to cope with this behavior so that we believe our transmission function is uniform to better than $23 \%$ at $E_{0}=10 \mathrm{eV}$ and $7 \%$ at $E_{0}=100 \mathrm{eV}$. The second normalization approach we utilized, again at each $E_{0}$, was to measure energy loss spectra at each chosen $\theta_{\mathrm{e}}$ and, in the manner just described, determine the elastic DCS directly from those energy-loss spectra. This second approach is relatively more time consuming, compared to method 1 , as energy loss spectra at all relevant $\theta_{\mathrm{e}}$ are now required. The efficacy here, however, is that the effective path-length correction factor $[26,27]$ cancels out in taking the ratio, and is thus not relevant with this approach. However, the analyzer transmission function issue remains open. Nonetheless, as noted earlier, the elastic DCSs we determined from the two normalization techniques, irrespective of $E_{0}$, were always consistent to within the uncertainties we cite. This gives us some confidence in the utility of our experimental measurement techniques and procedures. A summary of the current measured elastic DCSs and their uncertainties is given in Table I, with plots of those results and our new OP computations being found in Figs. 2 and 3 .

The uncertainties in our measured DCSs stem from a variety of contributions. As noted earlier, the stabilities of the electron and atomic beams are both better than $2 \%$ over the lifetime of a given experimental run. Despite the large dynamic range of the elastic intensity over the $\theta_{\mathrm{e}}$ we probed (see Table I), the statistical uncertainties in our angular distributions were rarely worse than $30 \%$ and only then at the larger scattering angles. To place the angular distributions on an absolute scale, we carry over the intrinsic uncertainty on the earlier ${ }^{4} P_{1 / 2}$ inelastic DCS from Marinković et al. [24] at the normalization angle, the uncertainty in our effective path-length correction factor $(<5 \%)$, the uncertainty of $\sim 1 \%$ in our energy and angular calibrations, and the uncertainty of $\sim 23 \%$ at $10 \mathrm{eV}$ decreasing to $\sim 7 \%$ at $100 \mathrm{eV}$ on our analyzer transmission functions associated with the energyloss measurements and our determination of the elastic to inelastic ratios. When combining all these contributions in quadrature, we found that the overall uncertainties on our elastic DCSs typically lay in the range $15 \%-55 \%$, with the exact uncertainties at each $E_{0}$ and $\theta_{\mathrm{e}}$ being found in Table I.

Having determined our elastic DCSs, we now need to extrapolate them to $\theta_{\mathrm{e}}=0^{\circ}$ and $180^{\circ}$, perform an interpolation, and then undertake the appropriate integrations in order to derive the elastic ICSs and MTCSs at each energy. Three approaches were utilized to achieve that aim. In the first a "by eye" empirical procedure was used, while in the second the shape of our OP theory results, after allowance was made for the monomer or dimer mixture in our experimental beam, was employed. Finally, the complex phase-shift analysis of Allen and co-workers $[32,33]$ provided our final self-consistency check. In all cases the ICSs and MTCSs we obtained, from each of the aforementioned approaches, were found to be consistent with one another to within our uncertainty estimate 
TABLE I. Differential cross sections for elastic electron scattering from bismuth. The last two rows are the integrated elastic ICS $\left(Q_{\mathrm{I}}\right)$ and momentum transfer cross sections $\left(Q_{\mathrm{MTCS}}\right)$ in units of $10^{-20} \mathrm{~m}^{2}$. The absolute uncertainties are indicated in parentheses.

\begin{tabular}{|c|c|c|c|c|c|c|}
\hline \multirow{2}{*}{$\begin{array}{l}\text { Scattering } \\
\text { angle } \\
\text { (degrees) }\end{array}$} & \multicolumn{6}{|c|}{$\operatorname{DCS}\left(10^{-20} \mathrm{~m}^{2} \mathrm{sr}^{-1}\right)$} \\
\hline & $10 \mathrm{eV}$ & $20 \mathrm{eV}$ & $40 \mathrm{eV}$ & $60 \mathrm{eV}$ & $80 \mathrm{eV}$ & $100 \mathrm{eV}$ \\
\hline 10 & $135(46)$ & $145(22)$ & $45.5(7.7)$ & $31.3(4.9)$ & $19.8(3.1)$ & $17.1(2.8)$ \\
\hline 20 & $60(21)$ & $38.2(5.9)$ & $4.89(0.85)$ & $3.03(0.17)$ & $2.69(0.42)$ & $2.62(0.43)$ \\
\hline 30 & $25.1(8.6)$ & 7.93(1.19) & $0.705(0.141)$ & $1.08(0.48)$ & $1.48(0.23)$ & $1.85(0.30)$ \\
\hline 40 & $10.3(3.5)$ & $1.65(0.26)$ & $0.349(0.080)$ & $0.86(0.14)$ & $0.99(0.15)$ & $1.01(0.17)$ \\
\hline 50 & $4.9(1.7)$ & $0.665(0.102)$ & $0.722(0.145)$ & $0.500(0.081)$ & $0.501(0.078)$ & $0.310(0.051)$ \\
\hline 60 & $2.81(0.96)$ & $0.827(0.128)$ & $0.968(0.185)$ & $0.259(0.043)$ & $0.242(0.038)$ & $0.189(0.031)$ \\
\hline 70 & $1.54(0.53)$ & $0.836(0.128)$ & $0.601(0.122)$ & $0.171(0.029)$ & $0.195(0.031)$ & $0.156(0.026)$ \\
\hline 80 & $1.02(0.35)$ & $0.470(0.073)$ & $0.265(0.063)$ & $0.142(0.024)$ & $0.251(0.039)$ & $0.170(0.028)$ \\
\hline 90 & $0.57(0.19)$ & $0.188(0.030)$ & $0.141(0.042)$ & $0.142(0.024)$ & $0.247(0.039)$ & $0.209(0.035)$ \\
\hline 100 & $0.48(0.16)$ & $0.224(0.036)$ & $0.575(0.117)$ & $0.177(0.030)$ & $0.229(0.036)$ & $0.228(0.038)$ \\
\hline 110 & $0.56(0.19)$ & $0.374(0.059)$ & $1.09(0.19)$ & $0.304(0.049)$ & $0.228(0.036)$ & $0.102(0.017)$ \\
\hline 120 & $0.72(0.25)$ & $0.351(0.055)$ & $1.27(0.24)$ & $0.374(0.061)$ & $0.183(0.029)$ & $0.0819(0.0137)$ \\
\hline 130 & $0.94(0.32)$ & $0.232(0.037)$ & $0.668(0.136)$ & $0.310(0.051)$ & $0.101(0.017)$ & $0.0455(0.0076)$ \\
\hline 140 & $1.20(0.41)$ & $0.317(0.050)$ & $0.509(0.107)$ & $0.121(0.021)$ & $0.0391(0.0063)$ & $0.0916(0.0153)$ \\
\hline 150 & $1.40(0.76)$ & $1.16(0.18)$ & $0.731(0.144)$ & $0.134(0.023)$ & $0.0730(0.0116)$ & $0.205(0.034)$ \\
\hline$Q_{\mathrm{I}}$ & $89.2(26.8)$ & $58.9(17.7)$ & $21.8(6.5)$ & $12.2(3.7)$ & $9.2(2.8)$ & $9.1(2.7)$ \\
\hline$Q_{\text {MTCS }}$ & $19.3(5.8)$ & $12.1(3.6)$ & $9.2(2.8)$ & $3.29(1.0)$ & $2.44(0.73)$ & $2.49(0.75)$ \\
\hline
\end{tabular}

of $\pm 30 \%$. A summary of our ICS and MTCS results is given in Table I, with the elastic ICS results also being plotted in Fig. 4.

\section{THEORETICAL DETAILS}

We have recently described our standard optical potential approach in our studies of the electron-beryllium [34] and electron-magnesium [35] scattering systems. All the generic details of our atomic OP method, including the forms of the static potential, polarization potential, and the exchange potential, that we use, were given in those papers [34,35] and so only the key points of this method are summarized here. The electron-atom interaction is described by a local complex potential given by

$$
V(r)=V_{\mathrm{s}}(r)+V_{\mathrm{ex}}(r)+V_{\mathrm{pol}}(r)+i V_{\mathrm{abs}}(r),
$$

where the real part comprises the following three terms. $V_{\mathrm{s}}$ is the static term derived from a Hartree-Fock calculation [36] of the atomic charge distribution. $V_{\mathrm{ex}}$ is an exchange term which accounts for the indistinguishability of the incident and target electrons; it is given by the semiclassical energydependent formula derived by Riley and Truhlar [37]. $V_{\mathrm{pol}}$ is a polarization potential for the long-range interactions which depend on the target dipole polarizability, in the form given by Zhang et al. [38].

The imaginary absorption potential accounts for the electronically inelastic scattering events. It is based on the quasifree model by Staszewska et al. [39] but incorporates some improvements to the original formulation, such as the inclusion of screening effects, local velocity corrections, and the description of the electron indistinguishability [40] leading therefore to a model which provides a realistic approximation for electron-atom scattering over a broad energy range [41].

In this study, however, different forms of the static potential and polarization potential were also applied, with the cross section results from using those new potentials being denoted here as OP2. In this latter case, a relativistic formulation was used for the calculation of the static potential. The spin-orbit interaction is then included and the three $6 \mathrm{p}$ electrons give rise to the five states $6 \mathrm{p}^{3}{ }^{4} S_{3 / 2}, 6 \mathrm{p}^{3}{ }^{4} D_{3 / 2,5 / 2}$, and $6 \mathrm{p}^{3}{ }^{4} P_{1 / 2,3 / 2}$. A linear combination of the wave functions corresponding to these five states was then used in a five-state Dirac-Fock multiconfiguration calculation [42] to determine the groundstate wave function of $\mathrm{Bi}$. The static potential of $\mathrm{Bi}$ was then determined in the usual manner [see Eq. (9) in Chen et al. [43]].

The asymptotic form of the polarization potential can be expressed analytically in terms of the static multipole polarizabilities of the atom. However, there is no such analytic form for the polarization potential inside the charge density of the atom. In order to ensure that this potential goes to zero (or a small number) at the origin, one can use a parametrized form for this potential as was done in Eq. (4) in this region. However, in the polarized-orbital method for closed sub-shell atoms $[44,45]$, there are no such adjustable parameters. Rather, one uses first-order perturbation theory to calculate adiabatically, the first-order correction to the atomic wave functions due to the charge of the incident electron at a distance $r$ from the origin. The polarization potential inside the atom is then given in terms of indefinite integrals involving the unperturbed Hartree-Fock orbitals $P_{n l}(r)$ and their first-order corrections $F_{n l}^{v v^{\prime}}(r)$ according to

$$
V_{\mathrm{p}}(r)=-2 \sum_{\substack{n l \\ v v^{\prime}}} \frac{1}{r} G_{v^{\prime} v}^{l^{\prime}} y_{\nu}\left(P_{n l}(r), F_{n l}^{v v^{\prime}} ; r\right),
$$

where the parameter $G_{v^{\prime} v}^{l^{\prime}}$ is given in terms of $3-j$ symbols by

$$
G_{v^{\prime} v}^{l^{\prime}}=\frac{(2 l+1)(2 v+1)}{\left(2 v^{\prime}+1\right)}\left(\begin{array}{c}
l v v^{\prime} \\
000
\end{array}\right)^{2} .
$$



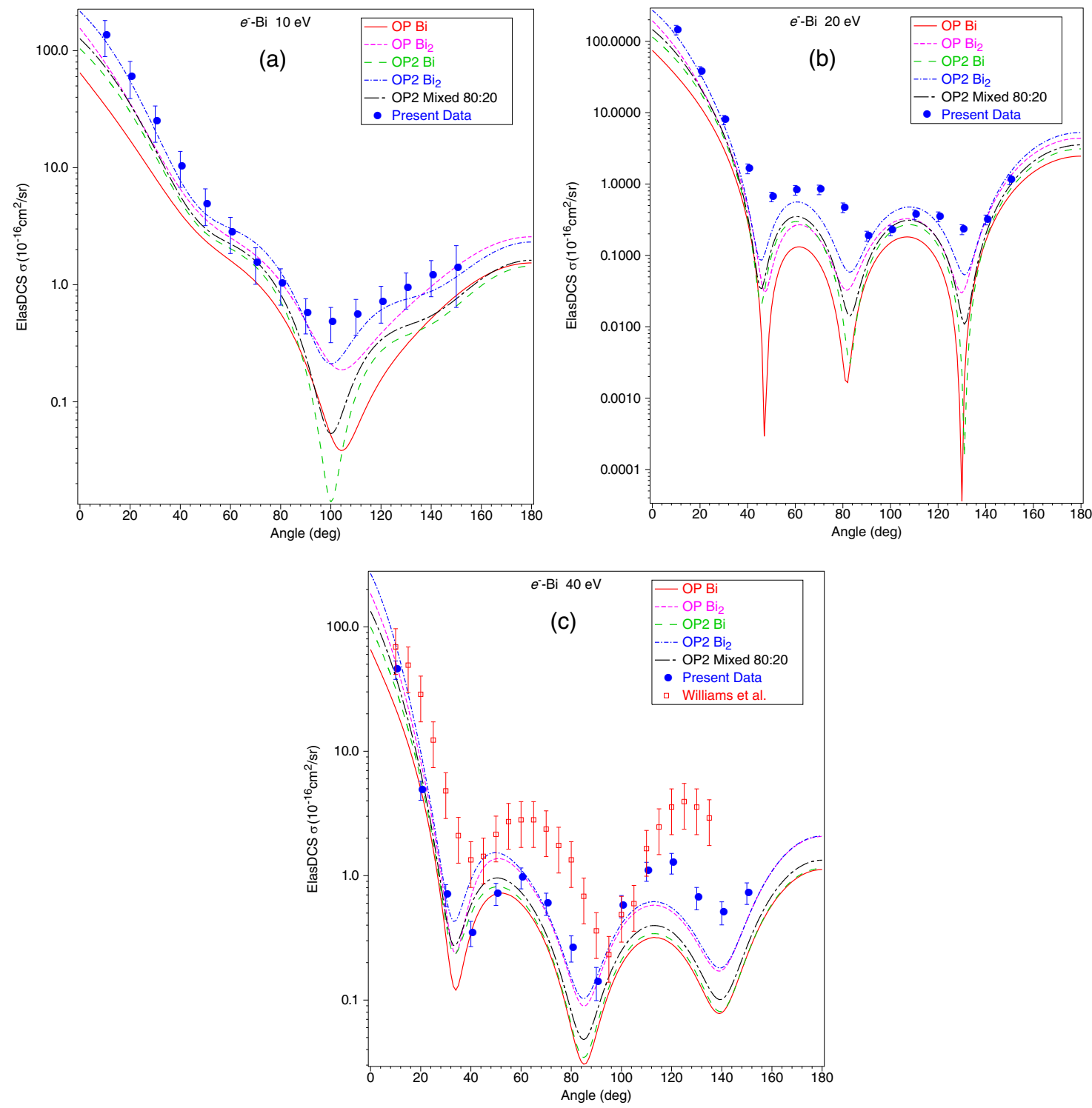

FIG. 2. Differential elastic cross sections $\left(10^{-16} \mathrm{~cm}^{2} / \mathrm{sr}\right.$ ) for electron scattering from bismuth at (a) $10 \mathrm{eV}$, (b) $20 \mathrm{eV}$, and (c) $40 \mathrm{eV}$. The present measurements $(\bullet)$ and those of Williams et al. [19] ( $\square$ ) are compared against the current OP results for $\mathrm{Bi}(-)$ and $\mathrm{Bi}_{2}(-----)$, our $\mathrm{OP} 2$ results for $\mathrm{Bi}(--)$ and $\mathrm{Bi}_{2}(-\cdot-\cdot)$, and our mixed monomer and dimer OP2 results $(---)$. See text for further details.

Here the variables $n l$ are the usual principal and angular momentum quantum numbers and $v$ is the order of the multipole; $v^{\prime}$ is just a dummy summation variable. Furthermore, the first-order corrections $F_{n l}^{v v^{\prime}}(r)$ are solutions of the integrodifferential Eq. (12) of Ref. [44].

As a consequence, this polarization potential actually reflects the internal charge density structure of the atom. In order to improve upon the adiabatic nature of this polarization potential, a nonadiabatic correction to this potential, called the dynamic distortion potential, can also be included [46].

The polarized-orbital method also yields reliable estimates of the atomic static multipole polarizabilities of the atom $[47,48]$. However, the values of these static polarizabilities are only influenced by the outermost two to three $n$ shells of the atom where $n$ is the principal quantum number. It is also the contribution of these outermost atomic $n$ shells to the polarization potential which are the most impor- tant in a scattering calculation since, for relatively small $r$, the total scattering potential is dominated by the static potential.

In the case of $\mathrm{Bi}$, which has an outer electron configuration of $6 \mathrm{p}^{3}$, the overall charge density of this atom will be very similar to that of the closed-shell atom $\mathrm{Rn}\left(6 \mathrm{p}^{6}\right)$ except for the outermost $n=5$ and 6 shells. Consequently, their polarization potentials will also be very similar, except for the outermost distances $r$ inside the atom. Thus, in order to approximate an $a b$ initio polarization potential for $\mathrm{Bi}$, we have scaled the polarization potential of Rn by the ratio of their dipole polarizabilities. This radon polarization potential included the first eight static multipoles (i.e., $v=1-8$ ) as well as the corresponding dynamic distortion potential defined in Eq. (23) of Ref. [46]. This overall procedure has proved highly successful in the treatment of electron and positron scattering from other atoms and molecules [49]. 

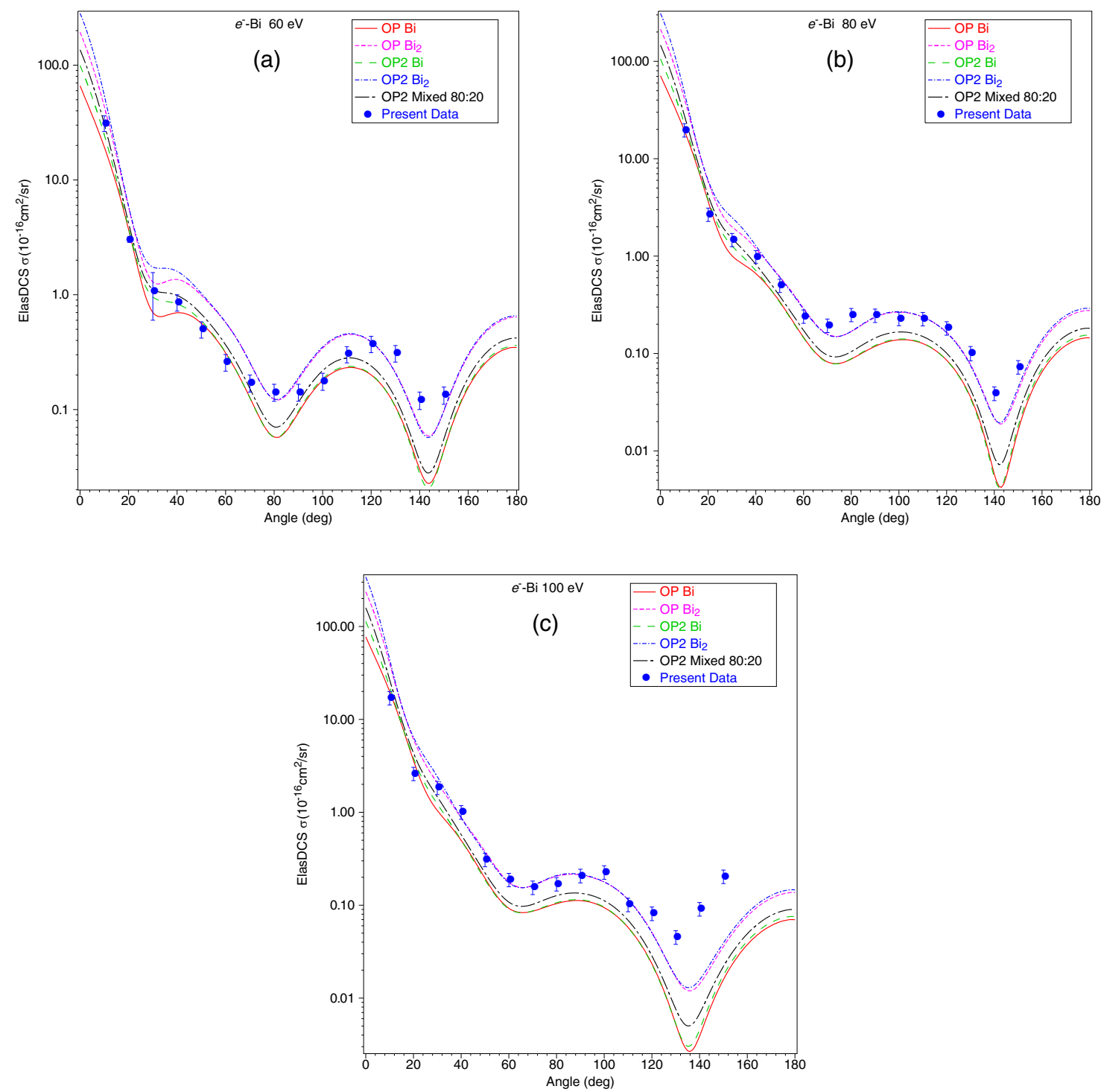

FIG. 3. Differential elastic cross sections $\left(10^{-16} \mathrm{~cm}^{2} / \mathrm{sr}\right.$ ) for electron scattering from bismuth at (a) $60 \mathrm{eV}$, (b) $80 \mathrm{eV}$, and (c) $100 \mathrm{eV}$. The present measurements $(\bullet)$ are compared against the current $\mathrm{OP}$ results for $\mathrm{Bi}(-)$ and $\mathrm{Bi}_{2}(-----)$, our $\mathrm{OP} 2$ results for $\mathrm{Bi}(--)$ and $\mathrm{Bi} 2(-\cdot-\cdot)$, and our mixed monomer and dimer OP2 results ( - - - ). See text for further details.

As a result of the experimental beam containing a component $(\sim 20 \%)$ of $\mathrm{Bi}_{2}$, we also needed to calculate the cross sections for bismuth's molecular dimer. This was achieved with our independent atom method complemented with the screening corrected additivity rule [50,51] and including interference effects (IAM-SCAR+I) [52]. In the independent atom method (IAM) with the additivity rule (AR), the dimer cross sections would simply be twice those of the monomer cross sections (at each $E_{0}$ and $\theta_{\mathrm{e}}$ ). This representation is clearly unphysical at lower incident electron energies. To account for that Blanco and colleagues [50-52] introduced corrections to consider the molecular geometry to account for the overlapping of the atomic cross sections, and additionally it now allows for interference effects [52]. This is the socalled IAM-SCAR+I approach which has been successfully employed, on many molecular systems, for some years now (see, e.g., Ref. [53] and references therein).

\section{RESULTS AND DISCUSSION}

In Fig. 1 we show some typical results for electron energyloss spectra from the current study. For atomic systems, particularly for lower $n$ (principal quantum number) values of the valence shell, we would anticipate to see a series of well-resolved lines. In this case, however, the picture is somewhat complicated by the presence of an appreciable $\mathrm{Bi}_{2}$ dimer contribution $(\sim 20 \%)$ to the measured spectra. In particular, features $\mathrm{B}, \mathrm{C}$, and $\mathrm{D}$, which do not correspond to any known atomic transitions in $\mathrm{Bi}$, are highlighted in Fig. 1. Note that there is also presumably a vibrational progression associated with the molecular electronic states denoted by B, $\mathrm{C}$, and D and with the elastic peak. The two most important points we can glean from Fig. 1 are that the elastic peak is well resolved from all the inelastic features, and that the important $6 \mathrm{p}^{2} 7 \mathrm{~s}{ }^{4} P_{1 / 2}$ inelastic peak (which is used in the normalization process) is also quite well resolved from its 


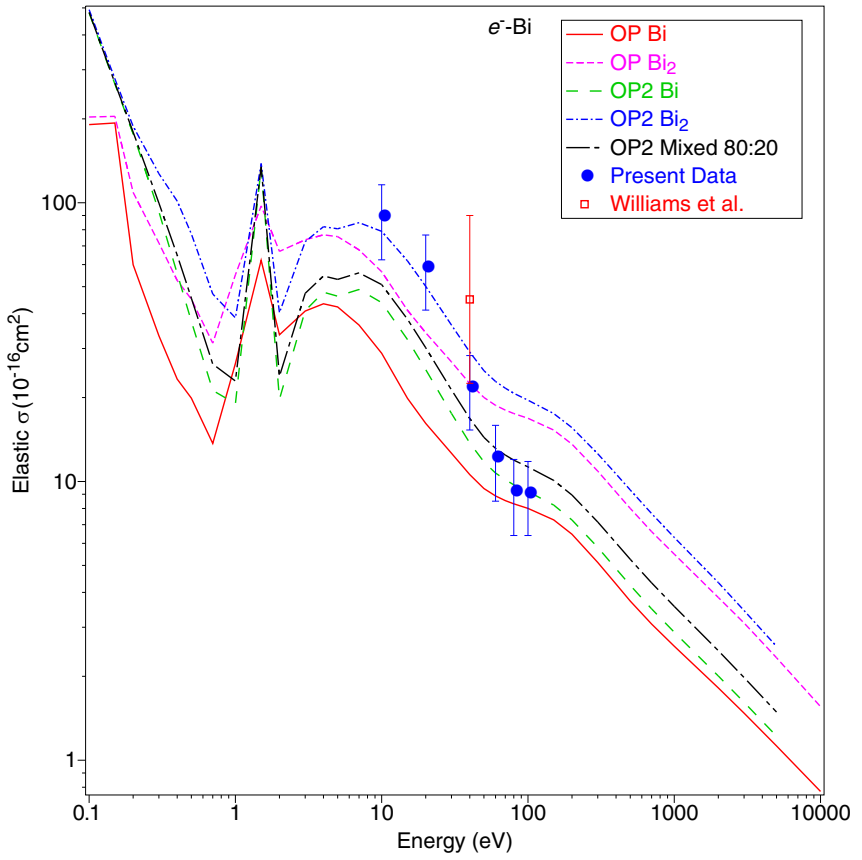

FIG. 4. Integral elastic cross sections $\left(10^{-16} \mathrm{~cm}^{2}\right)$ for electron scattering from bismuth. The present measurements $(\bullet)$ and that of Williams et al. [19] ( $\square$ ) are compared against the current OP results for $\mathrm{Bi}(-)$ and $\mathrm{Bi}_{2}(----)$, our OP2 results for $\mathrm{Bi}(--)$ and $\mathrm{Bi}_{2}$ $(-\cdot-\cdot)$, and our mixed monomer and dimer OP2 results (- - - ). See text for further details.

nearest neighbors or could be spectrally deconvolved [54] from its nearest neighbors if required.

In Fig. 2 for $10 \mathrm{eV}, 20 \mathrm{eV}$, and $40 \mathrm{eV}$, and Fig. 3 for $60 \mathrm{eV}, 80 \mathrm{eV}$, and $100 \mathrm{eV}$, we present our experimental and theoretical differential cross section results for elastic electron scattering from bismuth. Specifically, our theory results show the Bi DCS calculated within our atomic optical potential approach with our usual scattering potentials (denoted as OP) and with our modified scattering potentials (denoted as OP2; see Sec. III for more details). In addition, corresponding IAM-SCAR+I Bi 2 DCS are also shown, calculated in each case for the different atomic OP results, and denoted as OP $\mathrm{Bi}_{2}$ or OP2 $\mathrm{Bi}_{2}$, respectively. Differences between our OP Bi and OP2 Bi DCSs were subtle (see Figs. 2 and 3), and were mainly seen in the magnitudes of the calculated cross sections at the lower incident electron energies. However, the shapes of the OP and OP2 monomer DCSs were very similar at all the energies probed. A similar scenario is also observed when we compare the dimer $\mathrm{OP} \mathrm{Bi}_{2}$ and $\mathrm{OP} 2 \mathrm{Bi}_{2}$ DCSs. Interestingly, and irrespective of whether we are considering the $\mathrm{OP} \mathrm{Bi}$ and OP $\mathrm{Bi}_{2}$ DCSs or OP2 Bi and OP2 $\mathrm{Bi}_{2}$ DCSs, the effect of the molecular application is to relatively increase the magnitude of the DCSs and to somewhat "fill-in" the pronounced angular structure observed at all energies in the differential cross sections. This latter behavior is entirely due to the screening coefficients and interference terms, that have been incorporated into our molecular cross section calculations in order to extend the validity of our approach to incident electron energies below $100 \mathrm{eV}$ where the additivity rule fails. Note that both these corrections to the additivity rule contribute to the observed "fill-in" effect, as was explicitly checked by repeating our computations with them included and excluded, as required. Further note that we consider the polarization and static potentials that we employed in our OP2 computations to be more accurate than those we typically use in our OP atomic method. As a consequence, in the discussion that follows comparing our theoretical and measured DCSs in more detail, it is the mixed beam $(80 \%-20 \%)$ OP2 results that best mimic those DCSs measured experimentally. These "mixed" theory cross sections are denoted as OP2 Mixed 80:20 in all the plots of Figs. 2 and 3.

Considering Fig. 2 in more detail, then at each energy $(10 \mathrm{eV}, 20 \mathrm{eV}$, and $40 \mathrm{eV}$ ) we find good qualitative accord (i.e., in terms of the shapes of the elastic angular distributions) between our measured data and our OP2 "mixed" calculated cross section. In terms of the absolute magnitudes, however, the $10 \mathrm{eV}$ and $20 \mathrm{eV}$ measured data tend to be somewhat higher in value compared to our preferred theoretical result, across all $\theta_{\mathrm{e}}$, with the discrepancy between them being a little more pronounced at $20 \mathrm{eV}$ compared to $10 \mathrm{eV}$. By $40 \mathrm{eV}$ that trend in the comparison of the absolute magnitudes at lower energies has changed, with pretty good quantitative agreement between the experimental and "mixed" theoretical DCSs now being found for $\theta_{\mathrm{e}} \lesssim 60^{\circ}$. Above that scattered electron angle, however, the theory once again predicts a DCS that is smaller in magnitude compared to that measured experimentally. At $40 \mathrm{eV}$ [see Fig. 2(c)] we can also compare our DCS against the only other reported values in the literature from Williams et al. [19]. While the overall shapes of the experimental angular distributions are observed to be in quite fair accord, there is a large mismatch in their absolute values. As noted earlier it is well known that the pioneering early work with metal vapors at JPL has not stood the test of time, so that the present results supercede those from Williams et al. [19].

The trend in the comparison, between our experimental DCSs and "mixed" theory computations, that we have just discussed at $40 \mathrm{eV}$ is equally applicable to our results at $60 \mathrm{eV}$, $80 \mathrm{eV}$, and $100 \mathrm{eV}$ (see Fig. 3). Namely, at the more forward scattered electron angles there is good quantitative accord between measurement and the "mixed" theory calculation but at higher $\theta_{\mathrm{e}}$ the experimental data is somewhat stronger in magnitude than the theory. For all of the cross sections in Figs. 2 and 3, we believe that the observed discrepancies between our measured and calculated results are, at least in part, due to the "flux competition" in the theory between the open elastic, discrete inelastic, and ionisation channels at a given incident electron energy. Assuming our measured DCSs are in fact correct, then Figs. 2 and 3 suggest that more flux is going into the discrete inelastic and ionization channels, compared to the elastic channel, than should be the case. However, it is worth noting that the elastic DCSs at $40 \mathrm{eV}, 60 \mathrm{eV}$, and $100 \mathrm{eV}$, in particular, all have very small magnitudes at middle and backward electron-scattering angles. Therefore, it would only require a very small misapportionment of flux into the discrete inelastic and ionization channels to lead to what we find in Figs. 2(c) and 3. This highlights just how challenging these sorts of computations are, so that the level of accord that we achieve here between the "mixed" theory and experiment in Figs. 2 and 3 is actually pretty good. Note that the effects of flux competition were previously elucidated upon by us in 
our recent work with electron scattering from zinc [23], and are also found in electron-molecule collision systems [55].

One of the key features from the present study is the strong oscillatory nature of the angular distributions in Figs. 2 and 3. The oscillatory nature of any elastic DCS arises from the interference, both constructive and destructive, between the various partial waves. At very low energies where the $s$ wave dominates, the DCS is almost effectively structureless. At higher energies, where the $p$ wave becomes important, the DCS will have a single minimum [related to the zero in $P_{1}(\cos \theta)$ ]. Similarly, as the energy further increases and the $d$ wave becomes important the angular distribution of the DCS can have two minima [related to the two zeros of $P_{2}(\cos \theta)$ ]. One sometimes gets three minima, but this is rare as the higher order phase shifts are relatively small and primarily contribute to the magnitude and not the structure of the DCS. While not being strictly relevant to our Bismuth results between $10-100 \mathrm{eV}$, for completeness we also note that if there is a resonance structure, due to a particular phase shift going through $\pi / 2$ radians, then the angular structure of the DCS can be dominated by the structure of the corresponding Legendre polynomial.

At the foot of Table I and in Fig. 4 we present our derived elastic integral cross sections for electron scattering from bismuth. Also plotted in Fig. 4 are the results of our Bi OP and OP2 computations, $\mathrm{Bi}_{2}$ OP and OP2 IAM-SCAR+I computations, and our preferred OP2 ICS results that mimic the mixed $80 \%$ monomer and $20 \%$ dimer experimental-beam composition. The first point we can glean from Fig. 4 is the existence of a strong low-energy resonance feature at around $1.4 \mathrm{eV}$ in both our OP and OP2 Bi calculations. While our atomic optical potential method has been successfully benchmarked against corresponding results from a sophisticated $B$-spline $R$-matrix method in atomic iodine [41], we would suggest that further relativistic convergent close-coupling and $B$-spline $R$-matrix calculations are required to confirm the existence of this feature. Similarly, we know of no experimental studies into negative-ion atomic resonances in $\mathrm{Bi}$ and certainly none are captured in the comprehensive review of Buckman and Clark [56]. Thus experimental verification of this low-energy feature (see Fig. 4) would also be very desirable.

In his seminal review, Schulz [57] noted that resonances in electron-atom scattering systems can arise from the temporary capture of the incident electron by excited states of the atom (so-called core-excited or Feshbach resonances) or by the ground state of the atom (so-called shape resonances). In the latter case the potential forms a penetrable barrier which traps the incident particle near the target, where that barrier is formed by the angular momentum of the electron. Thus for shape resonances we expect $p-, d-, f$-wave resonances but generally not $s$-wave resonances, since they have $l=0$ and thus produce no barrier. In the present case the ${ }^{2} D_{3 / 2}$ and ${ }^{2} D_{5 / 2}$ excited states of $\mathrm{Bi}$ at $1.4158 \mathrm{eV}$ and $1.915 \mathrm{eV}$, respectively, are very close in energy to the peak of the calculated structure $(\sim 1.5 \mathrm{eV})$ in Fig. 4. However, Schulz [57] also noted that Feshbach-type resonances are usually long lived so that their energy widths are narrow whereas the structure in Fig. 4 is rather broad $(\sim 0.3 \mathrm{eV})$ in width. On the other hand shape resonances typically have a shorter lifetime so that they have a wider energy profile, which is consistent with what we observe in Fig. 4. To unambiguously interpret this feature we have decomposed our calculated elastic ICS into the various scattering channels (i.e., $s$ wave $(l=0), p$ wave $(l=1), d$ wave $(l=2)$, and so on) that contribute to its overall profile. On doing this we clearly find that the origin of this structure is in the $l=2$ scattering channel, so that the observed lowenergy structure in Fig. 4 can be assigned as a $d$-wave shape resonance. Note that our preference for a shape-resonance assignment over a Feshbach resonance is simply based on the broad width of the observed feature.

In Fig. 4 we observe a good level of quantitative accord, to within the measurement uncertainties, between our experimental ICS and the OP2 "mixed" 80:20 theory ICS at all $E_{0} \geqslant 40 \mathrm{eV}$. Given our recent discussion at the DCS level, that latter observation might appear a little counterintuitive at first sight. However, it can be understood as follows. Most of the contribution to the integrand of the ICSs, even allowing for the $\sin \theta$ weighting factor, comes from the more forward electron-scattering angles of the DCSs and this is precisely where, between $40 \mathrm{eV}$ and $100 \mathrm{eV}$, the experimental DCSs are in largely good accord with our OP2 "mixed" ICS computations. At lower energies, however, the experimental ICSs are stronger in magnitude compared to our preferred theoretical result, although in this case that observation is entirely consistent with our earlier DCS-level discussion at $10 \mathrm{eV}$ and $20 \mathrm{eV}$. Nonetheless, the qualitative trend in the ICS versus energy behavior, for incident electron energies between 10 and $100 \mathrm{eV}$, between our measured and calculated data, is in good accord (see Fig. 4) with the quantitative differences between them being again (mainly) likely due to the "flux competition" effect.

\section{CONCLUSIONS}

We have reported on experimental and theoretical results for elastic electron scattering from bismuth, and in doing so we have significantly extended the available cross section data base for this scattering system. Strong interference effects, both constructive and destructive, in the scattering phase shifts describing the elastic collision process were clearly observed in our angular distributions, as were their energy dependence. In addition, through our OP and OP2 theoretical calculations and a comparison between them, the pivotal role the target polarization plays in the scattering dynamics here was manifest. Furthermore both those computations predicted the temporary capture of the incident low-energy electrons (at $\sim 1.5 \mathrm{eV}$ ) by the target, leading to a resonance enhancement in the magnitude of the integral elastic cross section, after autodetachment, at that energy. The width of this rather broad feature was found to be $\sim 0.3 \mathrm{eV}$, and we have assigned it to be a $d$-wave shape resonance. When allowance was made for the $\mathrm{Bi}_{2}$ dimer contribution in the experimental target beam, the overall comparison between our most accurate OP2 computation and the measured differential and integral cross sections is typically qualitatively good. Indeed at some energies we might characterize the level of accord between our experiments and calculations as being semiquantitative. Nonetheless it is clear that further experimental and theoretical investigations into this collision system are required, in particular relativistic-level $B$-spline $R$-matrix and convergent close-coupling calculations would be highly desirable. 


\section{ACKNOWLEDGMENTS}

This work was financially supported, in part, by the Spanish Ministerio de Ciencia, Innovación y Universidades (Project No. FIS2016-80440), CSIC (Project No. LINKA20085), the Australian Research Council (Projects No. DP160102787 and No. DP180101655), the Ministry of
Education, Science and Technological Development (Project No. OI171020) of the Republic of Serbia, and the Ministry of Scientific and Technological Development, Higher Education and Information Society (Project No. 19/6-020/961-67/18) of the Republic of Srpska, Bosnia, and Herzegovina. We thank Dr. L. Campbell for his help with some aspects of this paper.
[1] F. Kaussen, H. Geesmann, G. F. Hanne, and J. Kessler, J. Phys. B 20, 151 (1987).

[2] R. Haberland and L. Fritsche, J. Phys. B 20, 121 (1987).

[3] Neerja and A. N. Tripathi, Eur. Phys. J. D 13, 5 (2001).

[4] B. N. G. Guthrie, Astroph. Space Sci. 15, 214 (1972).

[5] J. M. Jacobs and M. M. Dworetsky, Nature (London) 299, 535 (1982).

[6] C. R. Colway, Observatory 107, 188 (1987).

[7] L. Campbell and M. J. Brunger, Int. Rev. Phys. Chem. 35, 297 (2016).

[8] L. Campbell and M. J. Brunger, Plasma Sources Sci. Technol. 22, 013002 (2013).

[9] L. Campbell and M. J. Brunger, Icarus 326, 162 (2019).

[10] L. Campbell and M. J. Brunger, Geophys. Res. Lett. 36, L03101 (2009).

[11] L. Campbell and M. J. Brunger, Geophys. Res. Lett. 37, L22104 (2010).

[12] P. Chiu and I. Shih, Nanotechnology 15, 1489 (2004).

[13] M. E. Toimil Molares, N. Chtanko, T. W. Cornelius, D. Debrev, I. Enculescu, R. C. Blick, and R. Neumann, Nanotechnology 15, S201 (2004).

[14] J. L. Schonfelder, Proc. Phys. Soc. 87, 163 (1966).

[15] M. Fink, J. C. Ingram, and A. C. Yates, J. Phys. B 3, 536 (1970).

[16] M. Fink and A. C. Yates, Atomic Data 1, 385 (1970).

[17] F. Blanco, A. Muñoz, D. Almeida, F. Ferreira da Silva, P. Limão-Vieira, M. C. Fuss, A. G. Sanz, and G. García, Eur. Phys. J. D 67, 199 (2013).

[18] R. Haug, Z. Phys. 215, 350 (1968).

[19] W. Williams, S. Trajmar, and D. G. Bozinis, J. Phys. B 8, L96 (1975).

[20] P. J. O. Teubner, J. L. Riley, M. J. Brunger, and S. J. Buckman, J. Phys. B 19, 3313 (1986).

[21] M. J. Brunger, J. L. Riley, R. E. Scholten, and P. J. O. Teubner, J. Phys. B 21, 1639 (1988).

[22] D. O. Brown, D. Cvejanović, and A. Crowe, J. Phys. B 36, 3411 (2003).

[23] B. P. Marinković, R. Panajotović, D. Šević, R. P. McEachran, G. García, F. Blanco, and M. J. Brunger, Phys. Rev. A 99, 062702 (2019).

[24] B. P. Marinković, B. Predojević, D. Šević, and V. Pejčev, J. Phys. B 49, 235203 (2016).

[25] B. P. Marinković, D. M. Filipović, V. Pejčev, and D. Šević, Int. J. Mass Spectrom. 271, 76 (2008).

[26] R. T. Brinkman and S. Trajmar, J. Phys. E 14, 245 (1981).

[27] M. J. Brunger and S. J. Buckman, Phys. Rep. 357, 215 (2002).

[28] A. N. Nesmeyanov, Vapour Pressure of Chemical Elements (Elsevier, Amsterdam, 1963).

[29] C. Barus, Phil. Mag. 29, 141 (1890).

[30] M. Knudson, Ann. Phys. 333, 999 (1909).

[31] I. Langmuir, Phys. Rev 2, 329 (1913).
[32] L. J. Allen, M. J. Brunger, I. E. McCarthy, and P. J. O. Teubner, J. Phys. B 20, 4861 (1987).

[33] M. J. Brunger, S. J. Buckman, L. J. Allen, I. E. McCarthy, and K. Ratnavelu, J. Phys. B 25, 1823 (1992).

[34] R. P. McEachran, F. Blanco, G. García, and M. J. Brunger, J. Phys. Chem. Ref. Data 47, 033103 (2018).

[35] R. P. McEachran, F. Blanco, G. García, P. W. Stokes, R. D. White, and M. J. Brunger, J. Phys. Chem. Ref. Data 47, 043104 (2018).

[36] R. D. Cowan, The Theory of Atomic Structure and Spectra (University of California Press, Berkeley, 1981).

[37] M. E. Riley and D. G. Truhlar, J. Chem. Phys. 63, 2182 (1975).

[38] X. Zhang, J. Sun, and Y. Liu, J. Phys. B: At. Mol. Opt. Phys. 25, 1893 (1992).

[39] G. Staszewska, D. W. Schwenke, D. Thirumalai, and D. G. Truhlar, Phys. Rev. A 28, 2740 (1983).

[40] F. Blanco and G. García, Phys. Rev. A 67, 022701 (2003).

[41] O. Zatsarinny, K. Bartschat, G. Garciá, F. Blanco, L. R. Hargreaves, D. B. Jones, R. Murrie, J. R. Brunton, M. J. Brunger, M. Hoshino, and S. J. Buckman, Phys. Rev. A 83, 042702 (2011).

[42] I. P. Grant, B. J. McKenzie, P. H. Norrington, D. F. Mayer, and N. C. Pyper, Comput. Phys. Commun. 21, 207 (1980).

[43] S. Chen, R. P. McEachran, and A. D. Stauffer, J. Phys. B 41, 025201 (2008).

[44] R. P. McEachran, D. L. Morgan, A. G. Ryman, and A. D. Stauffer, J. Phys. B 10, 663 (1977).

[45] R. P. McEachran, D. L. Morgan, A. G. Ryman, and A. D. Stauffer, J. Phys. B 11, 951 (1978).

[46] R. P. McEachran and A. D. Stauffer, J. Phys. B 23, 4605 (1990).

[47] R. P. McEachran, A. D. Stauffer, and S. Greita, J. Phys. B 12, 3119 (1979).

[48] E. Markiewicz, R. P. McEachran, and A. D. Stauffer, J. Phys. B 14, 949 (1981).

[49] L. Chiari, A. Zecca, S. Girardi, E. Trainotti, G. García, F. Blanco, R. P. McEachran, and M. J. Brunger, J. Phys. B 45, 215206 (2012).

[50] F. Blanco and G. García, Phys. Lett. A 317, 458 (2003).

[51] F. Blanco and G. García, Phys. Lett. A 330, 230 (2004).

[52] F. Blanco, L. Ellis-Gibbings, and G. García, Chem. Phys. Lett. 645, 71 (2016).

[53] F. Costa, L. Álvarez, A. I. Lozano, F. Blanco, J. C. Oller, A. Muñoz, A. Souza Barbosa, M. H. F. Bettega, F. Ferreira da Silva, P. Limão-Vieira, R. D. White, M. J. Brunger, and G. García, J. Chem. Phys. 151, 084310 (2019).

[54] L. Campbell, M. J. Brunger, P. J. O. Teubner, B. Mojarrabi, and D. C. Cartwright, Aust. J. Phys. 50, 525 (1997).

[55] M. J. Brunger, Int. Rev. Phys. Chem. 36, 333 (2017).

[56] S. J. Buckman and C. W. Clark, Rev. Mod. Phys. 66, 539 (1994).

[57] G. J. Schulz, Rev. Mod. Phys. 45, 378 (1973). 